\title{
Whistler mode propagation in the ionosphere in the presence of a longitudinal static electric field*
}

\author{
H. C. HSIEH \\ Electron Physics Laboratory, Department of Electrical Engineering, \\ The University of Michigan, Ann Arbor, Michigan, U.S.A.
}

(Received 15 October 1967)

\begin{abstract}
The effect of a longitudinal electrostatio field on whistler mode propagation has been studied in a region of the ionosphere where the influence of particle collisions and ion motion may be significant. As an illustration, the propagation in two cases of interest has been considered; one deals with the $E$-region in the neighborhood of $100 \mathrm{~km}$ height, and the other with the $F$-region in the neighborhood of $250 \mathrm{~km}$.

$I t$ is shown that the presence of a weak static electric field $\mathbf{E}_{0}$ tends to reduce the attenuation of the forward whistler mode when the magnetostatic field $\mathbf{B}_{0}$ and the electrostatio field $\mathbf{E}_{\mathbf{0}}$ are in the same direction. Moreover for a sufficiently large value of $\mathbf{E}_{0}$ the whistler mode may experience an amplification. On the other hand, when $\mathbf{E}_{0}$ and $\mathbf{B}_{0}$ are in opposite directions an increase in $\left|\mathbf{E}_{0}\right|$ tends to increase the attenuation of the wave. The variation of the attenuation constant, phase constant, and the phase velocity of the whistler mode with the signal frequency and with the electrostatic field is also discussed in detail.
\end{abstract}

\section{INTRODUCTION}

THE WHISTLER mode propagation in a warm collisionless electro-magneto-ionic medium has been studied recently by HsIEH (1967) using the coupled MaxwellVlasov equations. The method of analysis given by HsIEH (1967) is suitable for the analysis of whistler mode propagation in the magnetosphere but it is not adequate for consideration of the propagation in the lower ionosphere where the particle collision effects are known to play an important role. However this method of analysis can be easily extended so that the effect of collisions can be taken into account. This is done by using the Boltzmann equation with a properly assumed collision term instead of the Vlasov equation.

The purpose of the present paper is to discuss the effect of a longitudinal electrostatic field on whistler mode propagation in a region of the ionosphere where the effects of collisons and ion motion may be of significance.

In the present paper the following assumptions are made:

1. All quantities of interest are to be composed of two parts, a time-independent part and a time-varying part.

2. Small amplitude conditions are satisfied.

3. A one-dimensional analysis is applicable.

4. All time-dependent quantities have harmonic dependence of the form $\exp [j(\omega t-k z)]$, where $\omega$ and $k$ are the angular frequency and the propagation constant respectively; $t$ and $z$ are the time and spatial variables respectively.

* Sponsored by the National Aeronautics and Space Administration under Grant No. NsG 696. 
5. The effective collision frequency of electrons $\nu_{e}$ and that of positive ions $v_{i}$ are independent of the particle velocity.

6. Electrical neutrality is satisfied.

7. The equilibrium distribution function of electrons and ions has the form of a Maxwellian.

8. The electrostatic field $\mathbf{E}_{0}$, which is directed parallel to the magnetostatic field $\mathbf{B}_{0}$, is sufficiently weak so that the drift velocities of charged particles are much smaller than the phase velocity of the whistler mode.

\section{Dispersion Equatron}

Using the procedure of HsIEH (1967) and by combining the time-varying parts of the coupled Maxwell-Boltzmann equations the dispersion equation of the right-hand circularly polarized (whistler) mode for the system under consideration can be obtained and expressed as follows:

where

$$
1-\frac{c^{2} k^{2}}{\omega^{2}}=\frac{X_{1}}{W_{1}}\left(1+\frac{V_{1}{ }^{2} k_{1}{ }^{2}}{2 \omega^{2}} \frac{1}{W_{1}^{2}}\right)+\frac{X_{2}}{W_{2}}\left(1+\frac{V_{2}{ }^{2} k_{2}{ }^{2}}{2 \omega^{2}} \frac{1}{W_{2}^{2}}\right),
$$

$$
\begin{aligned}
& W_{1}=\left[\left(1-Y_{1}\right)-j Z_{1}\right], \quad k_{1}=\left(k+j K_{1}\right), \\
& W_{2}=\left[\left(1-Y_{2}\right)-j Z_{2}\right], \quad k_{2}=\left(k+j K_{2}\right), \\
& X_{1}=\left(\frac{\omega_{p}}{\omega}\right)^{2}, \quad Y_{1}=\left(\frac{\omega_{z}}{\omega}\right), \quad Z_{1}=\left(\frac{\nu_{s}}{\omega}\right), \\
& X_{2}=\left(\frac{\Omega_{p}}{\omega}\right)^{2}, \quad Y_{2}=\left(\frac{\Omega_{z}}{\omega}\right), \quad Z_{2}=\left(\frac{\nu_{i}}{\omega}\right), \\
& V_{1}=\left(\frac{2 K T_{e}}{m}\right)^{1 / 2}, \quad K_{1}=\left(\frac{e E_{0}}{K T_{e}}\right), \quad \omega_{z}=\left(\frac{e B_{0}}{m}\right), \\
& \omega_{\nu}=\left(\frac{e^{2} n}{m \varepsilon_{0}}\right)^{1 / 2}, \quad V_{2}=\left(\frac{2 K T_{i}}{M}\right)^{1 / 2}, \quad K_{2}=\left(\frac{-e E_{0}}{K T_{i}}\right),
\end{aligned}
$$

and

$$
\Omega_{z}=\left(\frac{-e B_{0}}{M}\right), \quad \Omega_{p}=\left(\frac{e^{2} N}{M \varepsilon_{0}}\right)^{1 / 2},
$$

in which $c=$ the speed of light in free space,

$\varepsilon_{0}=$ the dielectric constant of vacuum,

$e=$ the electron charge,

$m=$ the mass of electron,

$M=$ the mass of positive ion,

$T_{e}=$ the electron temperature,

$T_{i}=$ the ion temperature,

$K=$ the Boltzmann constant,

$v_{e}=$ the effective collision frequency of the electron and

$\nu_{i}=$ the effective collision frequency of the positive ion. 
It should be noted that equation ( 1 ) is a quadratic in $k$ and can conveniently be written as follows:

where

$$
\tilde{A}\left(\frac{\tilde{k}}{\beta_{0}}\right)^{2}+\tilde{B}\left(\frac{\tilde{k}}{\beta_{0}}\right)-\widetilde{C}=0
$$

in which

$$
\begin{aligned}
& \tilde{A}=\left[1+\tau X\left(\frac{1}{\tilde{W}_{1}^{3}}+\frac{\xi^{2}}{\theta} \frac{1}{\tilde{W}_{2}^{3}}\right)\right], \\
& \widetilde{B}=j 2 \delta X\left(\frac{1}{\widetilde{W}_{1}^{3}}-\frac{1}{\tilde{W}_{2}^{3}}\right), \\
& \widetilde{C}=\left[1-X\left(\frac{1}{\widetilde{W}_{1}}+\frac{\xi}{\widetilde{W}_{2}}\right)\right],
\end{aligned}
$$

$$
\begin{gathered}
\tilde{W}_{1} \equiv W_{1}, \quad \tilde{W}_{2} \equiv W_{2}, \quad X \equiv X_{1}, \quad Y \equiv Y_{1}, \\
\tau \equiv\left(\frac{K T_{e}}{m c^{2}}\right), \quad \delta \equiv\left(\frac{e E_{0}}{m c \omega}\right), \quad \xi \equiv\left(\frac{m}{M}\right), \quad \theta \equiv\left(\frac{T_{s}}{T_{i}}\right), \\
\beta_{0}=\left(\frac{\omega}{c}\right) .
\end{gathered}
$$

The symbol $\sim$ appearing in equation $(2)$ is introduced to emphasize the fact that the quantity under consideration is a complex quantity. In the present discussion the wave angular frequency $\omega$ is regarded as a real quantity and the propagation constant $\tilde{k}$ is regarded as a complex quantity, which can be written as

$$
\tilde{k}=(\beta-j \alpha),
$$

where $\alpha$ and $\beta$ are the amplitude and phase constants respectively. In view of the fact that the time and spatial dependence is assumed to be of the form

$$
\exp [j(\omega t-k z)]=\exp [-\alpha z+j(\omega t-\beta z)],
$$

a positive $\beta$ represents the forward wave, while a negative $\beta$ denotes the backward wave. It is apparent that positive $\alpha$ represents attenuation and negative $\alpha$ represents amplification of the wave. The two roots of equation (2a) give the propagation constants of the forward and backward waves. Once the system parameters are specified, equation (2a) can be solved for $\left(\tilde{k} / \beta_{0}\right)=\left[\left(\beta / \beta_{0}\right)-j\left(\alpha / \beta_{0}\right)\right]$. Thus the investigation of the behavior of roots of equation (2a) provides information with regard to the variation of the amplitude and phase of the whistler mode with the various system parameters.

\section{Whistler Mode Propagation in the $E$ - and $F$-Regions of the IONOSPHERE}

The $E$ - and $F$-regions of the ionosphere are regarded as consisting of a partially ionized gas in which electrons, positive ions and neutral particles may interact with each other. To study the propagation of a whistler mode in such regions a knowledge of the plasma parameter is required. For example, in order to calculate the net 
growth or decay in amplitude of a whistler wave which propagates through a slowly varying medium, knowledge of variation of such quantities as $n, N, \beta_{0}, E_{0}, T_{e}, T_{i}$, $v_{e}$ and $v_{i}$ along the whistler path is required.

A theoretical analysis made by NICOLET (1953) shows that the electron collision frequency in the ionosphere depends on the neutral particle concentration in $D$ - and $E$-regions and on the electron concentration in $F$-region. The collision frequency also depends on the temperature. For example, above the F2-layer, $v_{e i}$, the collision frequency of electrons with ions, can be approximately given by [NICoLET, 1953]

$$
v_{B i}=\left[34+8 \cdot 36 \log _{10}\left(T^{3 / 2} / n^{1 / 2}\right)\right] n T^{-3 / 2}
$$

where $n$ is the electron density and $T$ is the temperature of the plasma.

In the case of a plasma consisting of electrons and protons, the effective collision frequency of protons with electrons should be (see e.g., Frrraro and Plompton, 1961)

$$
\boldsymbol{v}_{p}=\left(m / m_{p}\right)^{1 / 2} \boldsymbol{v}_{\boldsymbol{e} i},
$$

where $m_{p}$ is the mass of the proton.

Some experimental data with regard to the profiles of number density and temperature of electron and positive ions in the ionosphere can be found in VALLEY (1965).

There is little information available in the literature as to the strength and spatial variation of the electrostatic field $E_{0}$ in the ionosphere. However, Mozer and BRUSTON (1967) recently reported sounding rockets measurements of $E_{0}=20 \mathrm{mV} / \mathrm{m}$ in the auroral ionosphere.

As an illustration of the method of analysis, the propagation characteristics of a whistler mode with frequency in the range between 1 and $20 \mathrm{kc} / \mathrm{sec}$, and with $\left|E_{0}\right|<10 \mathrm{mV} / \mathrm{m}$, are examined for two special cases of interest; Case I deals with the $E$-region in the neighborhood of height $h=100 \mathrm{~km}$, and Case II deals with the $F$-region in the neighborhood of $h=250 \mathrm{~km}$. The parameters chosen for this example are given below.

\begin{tabular}{ccccccccc}
\hline Cases & Region & $\begin{array}{c}\omega_{p} \\
\text { rad./seo }\end{array}$ & $\begin{array}{c}\omega_{z} \\
\text { rad./sec }\end{array}$ & $T_{a}^{\circ} \mathrm{K}$ & $T_{i}$ & $\begin{array}{c}\nu_{a} \\
\text { rad./sec }\end{array}$ & rad./sec & $\xi=\frac{m}{M} \cdot$ \\
\hline I & $h=100 \mathrm{~km}$ & $2.3 \times 10^{7}$ & $10^{7}$ & $270^{\circ}$ & $270^{\circ}$ & $3.14 \times 10^{5}$ & $0.28 \times 10^{3}$ & $4.0 \times 10^{-4}$ \\
II & $h=250 \mathrm{~km}$ & $4 \times 10^{7}$ & $8.8 \times 10^{8}$ & $2600^{\circ}$ & $1300^{\circ}$ & $3.14 \times 10^{4}$ & $1.83 \times 10^{2}$ & $0.34 \times 10^{-4}$ \\
\hline
\end{tabular}

The values of parameters $\omega_{p}, \omega_{z}, T_{e}$, and $T_{i}$ are taken from the data given by VALLEY (1965). The value of $\nu_{e}$ for Case $I$ is taken from SHKarofsky (1961), and the value of $\nu_{e}$ for Case II is derived with the aid of equation (4a). Moreover for both cases it is assumed that $\left(v_{i} / \nu_{e}\right)=(m / M)^{1 / 2}$. For Case II the positive ion under consideration is taken to be an oxygen ion.

Having specified the physical parameters of the region under consideration, for example, the variation of the amplitude and phase of a whistler mode with the wave angular frequency for different values of $E_{0}$ can be examined. The plots of $\alpha$ vs. $\omega$, $\beta$ vs. $\omega$ and $\left(v_{p h} / c\right)$ vs. $\omega$, with $v_{p h}$ denoting the phase velocity of the whistler mode are 


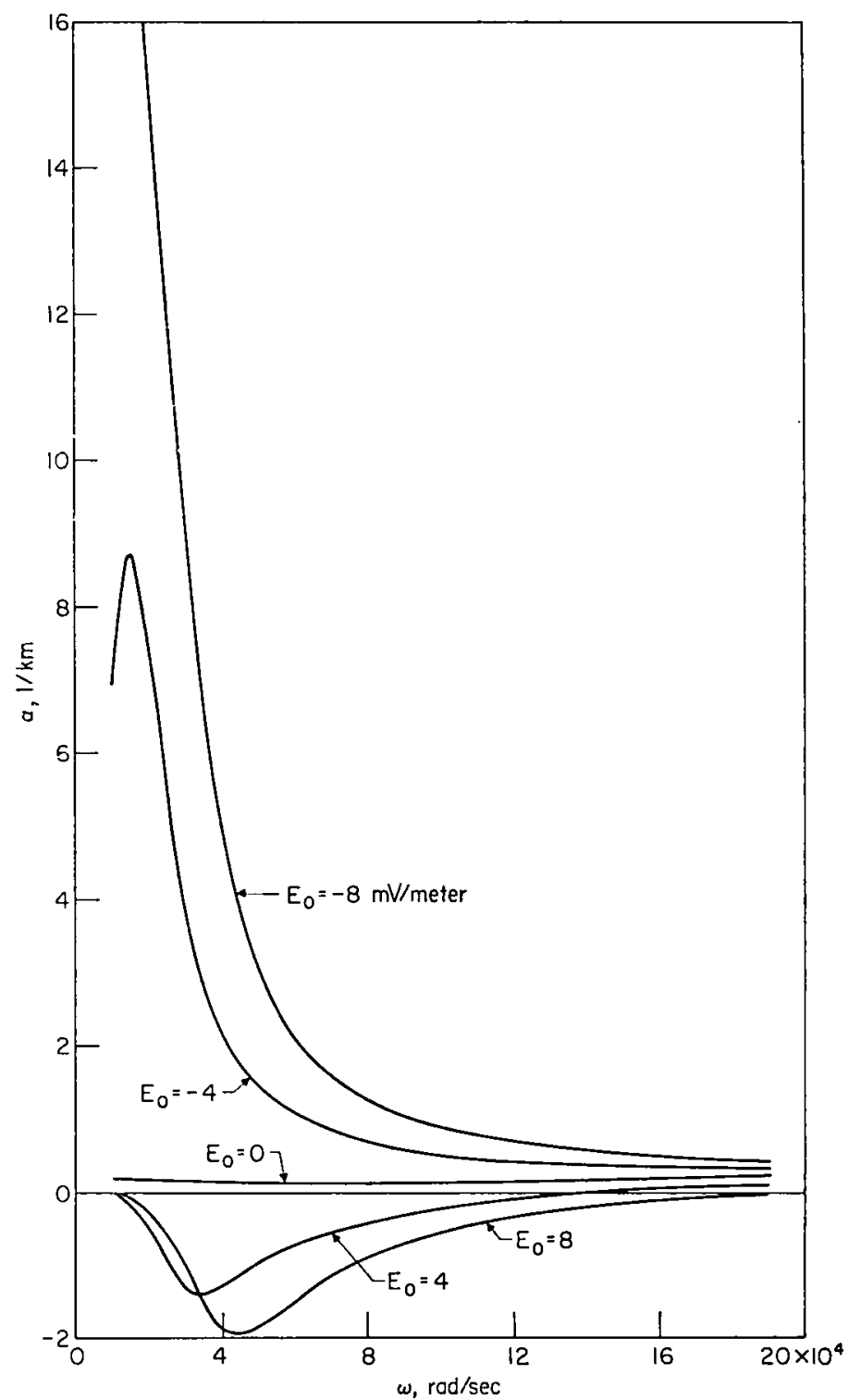

Fig. la. $\alpha$ vs. $\omega$ with $E_{0}$ as parameter for Case I.

shown in Figs. 1a, lb, and lc respectively for Case $I$, and the corresponding plots are shown in Figs. 2a, 2b, and 2c respectively for Case II.

\section{Discussion of Results}

It is well known that particle collisions lead to the attenuation of the whistler mode in the ionosphere. It is noted from Figs. la and 2a that when $E_{0}=0$ the attenuation constant $\alpha$ is essentially invariant with respect to the signal frequency. However, with the presence of an electrostatic field $\alpha$ does vary with the signal 


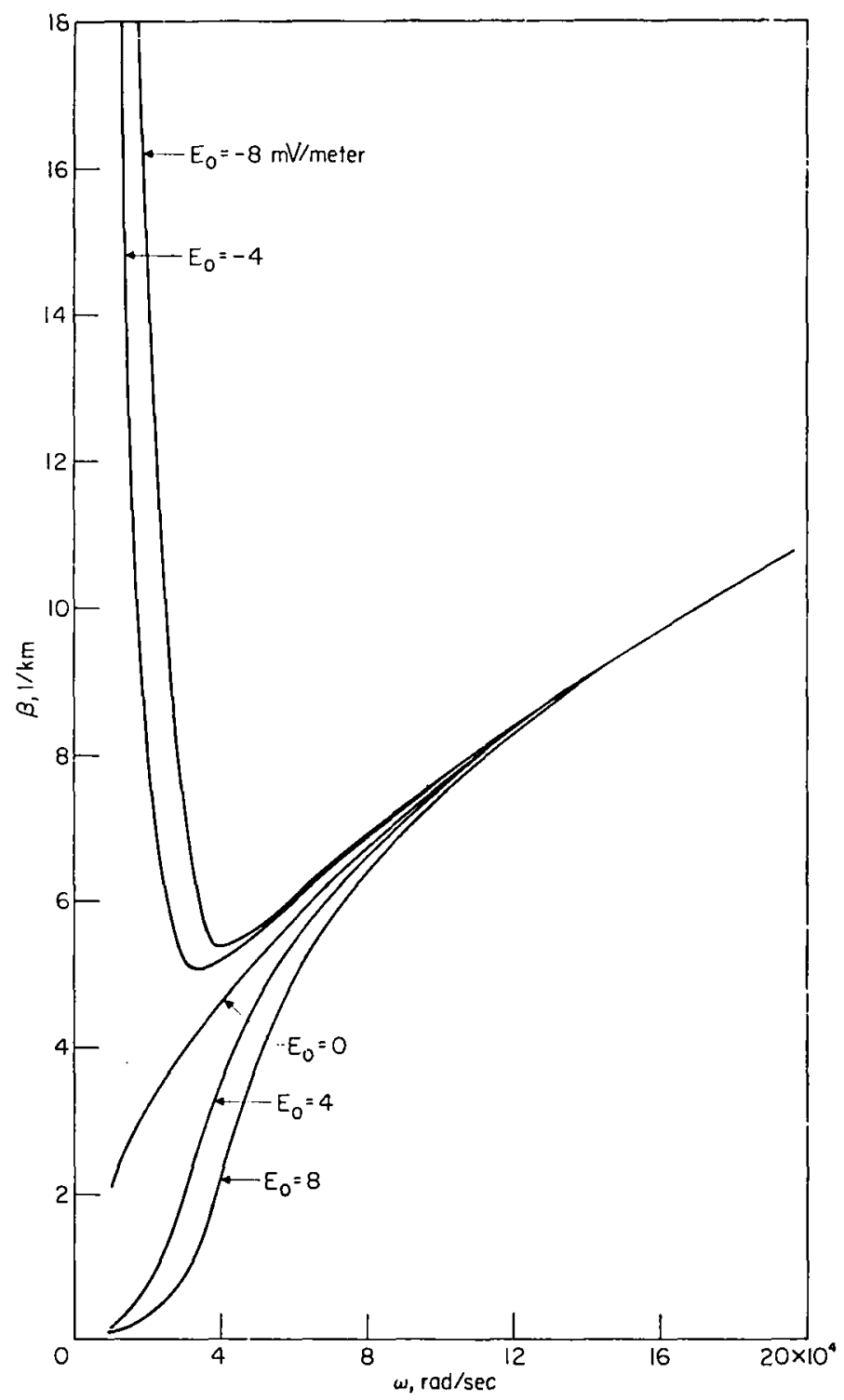

Fig. 1b. $\beta$ vs. $\omega$ with $E_{0}$ as parameter for Case 1 .

frequency. For $E_{0}>0$, the presence of $E_{0}$ leads to a reduction of the attenuation and if $E_{0}$ is sufficiently large then $\alpha$ may become negative; thus the wave is amplified. It should be noted that when $E_{0}>0$, for the parameters chosen, $\alpha$ is negative except in the range $\omega>15 \times 10^{4} \mathrm{rad} / \mathrm{sec}$ with $E_{0}=4 \mathrm{mV} / \mathrm{m}$ in Case $I$. This suggests that when $\mathbf{E}_{0}$ and $\mathbf{B}_{0}$ are both in the direction of the wave vector, the whistler mode experiences an amplification. Moreover when $E_{0}<0, \alpha$ is positive so that the wave experiences an attenuation when $\mathbf{E}_{0}$ and $\mathbf{B}_{0}$ are oppositely directed. $|\alpha|$ increases with $\left|E_{0}\right|$ in the range $\omega \geqq 4 \times 10^{4} \mathrm{rad} / \mathrm{sec}$ for Case $I$ and in the range $\omega \geqq 3 \times 10^{4}$ 


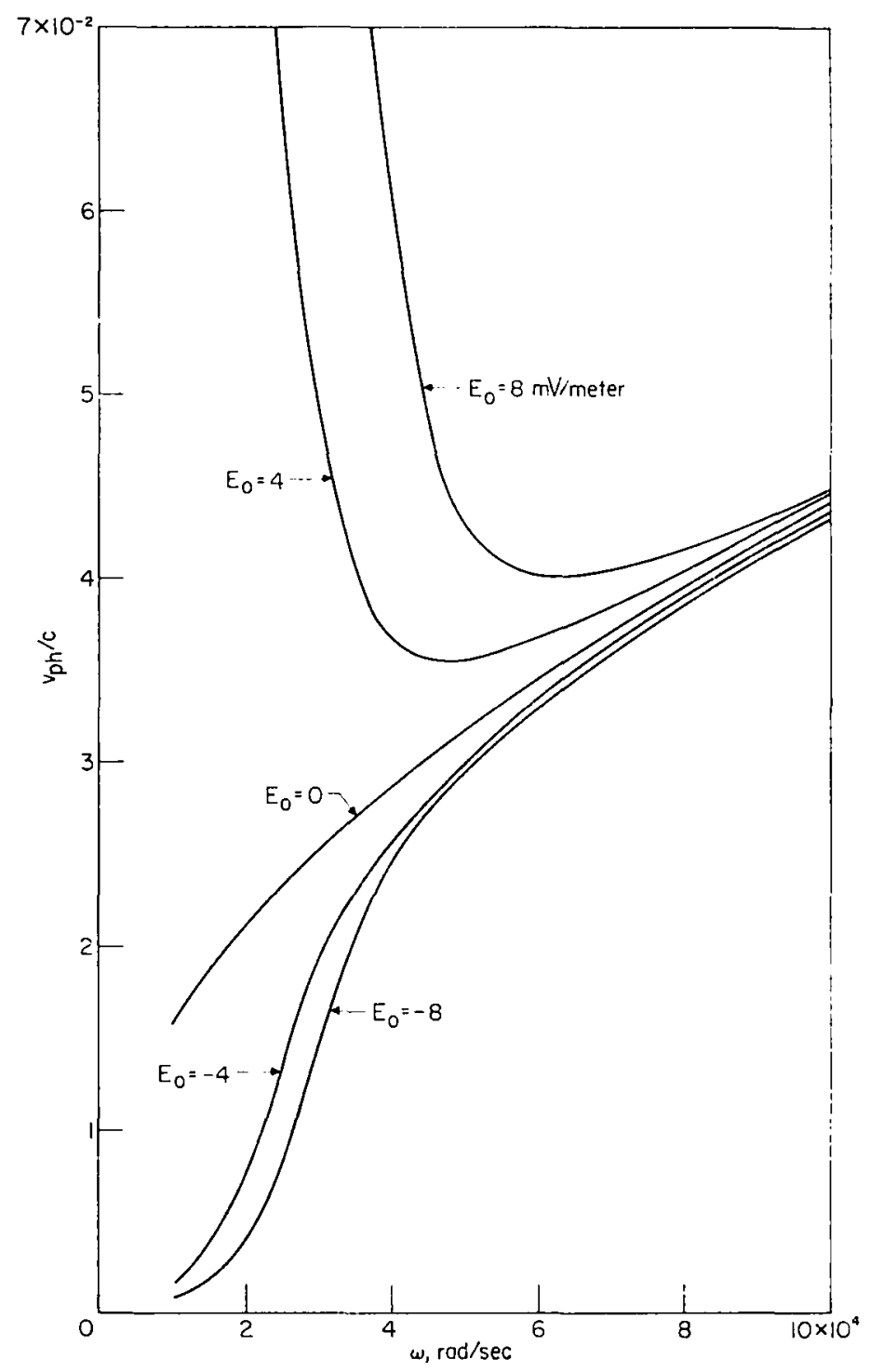

Fig. 1c. $\left(v_{p h} / c\right)$ vs. $\omega$ with $E_{0}$ as parameter for Case I.

$\mathrm{rad} / \mathrm{sec}$ for Case II. The maximum of $|\alpha|$ for both Cases I and II appears to lie somewhere in the low frequency range of the spectrum; $\omega \leqq 5 \times 10^{4} \mathrm{rad} / \mathrm{sec}$ for $\left|E_{0}\right|<8 \mathrm{mV} / \mathrm{m}$. The comparison of Fig. 1a with Fig. 2a shows that the extent of the effect of $E_{0}$ on $\alpha$ is greater in Case II than in Case I. However it should be noted that in Case $\mathrm{I}$ it is assumed that $T_{\mathrm{e}}=T_{i}$, whereas in Case II $T_{e}=2 T_{i}$.

On the other hand, from Figs. $1 \mathrm{~b}$ and $2 \mathrm{~b}$, it is observed that when $E_{0}=0$ the phase constant $\beta$ increases monotonically with the signal frequency. When $E_{0}>0$, the group velocity $v_{0} \equiv d \omega / d \beta$ is positive and varies with the signal frequency. However, when $E_{0}<0, d \omega / d \beta<0$ in the low frequency range and $d \omega / d \beta>0$ in the 


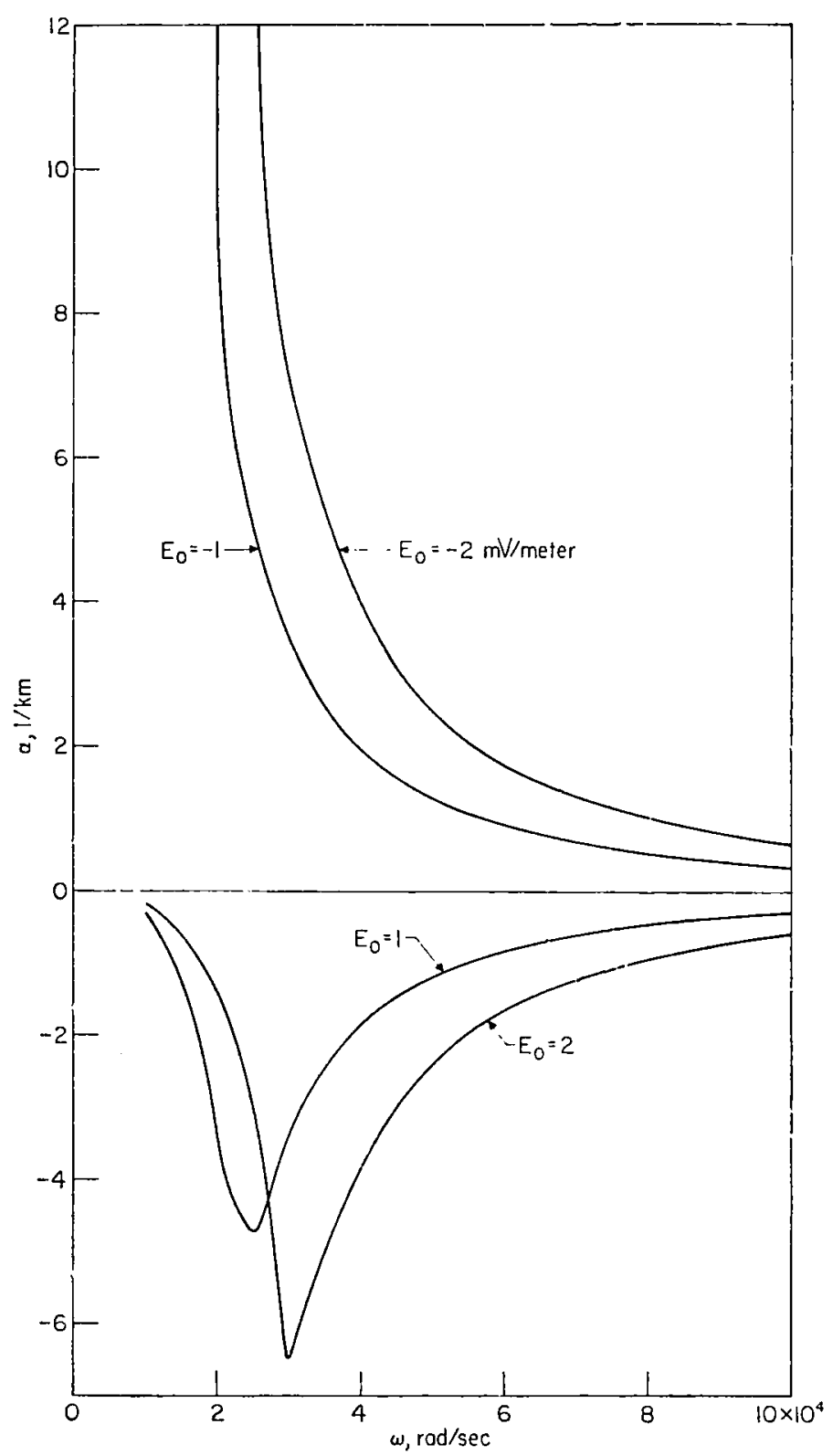

Fig. 2a. $\propto$ vs. $\omega$ with $E_{0}$ as parameter for Case $\Pi$.

high frequency range. Furthermore it is observed that in Case $I$, when $E_{0}>0$, the wavelength of the whistler mode increases with $\left|E_{0}\right|$ and when $E_{0}<0$, it decreases as $\left|E_{0}\right|$ increases. For Case $I I$ the wavelength increases with $\left|E_{0}\right|$ regardless of the algebraic signs of $E_{0}$. Finally it is observed that in Case I (see Fig. 1c) the phase velocity $v_{p h}$ of the whistler mode increases with $E_{0}$ when $E_{0}>0$ and decreases with $\left|E_{0}\right|$ when $E_{0}<0$. On the other hand, in Case II (see Fig. 2c), $v_{p h}$ increases with $\left|E_{0}\right|$ regardless of the algebraic sign of $E_{0}$. 


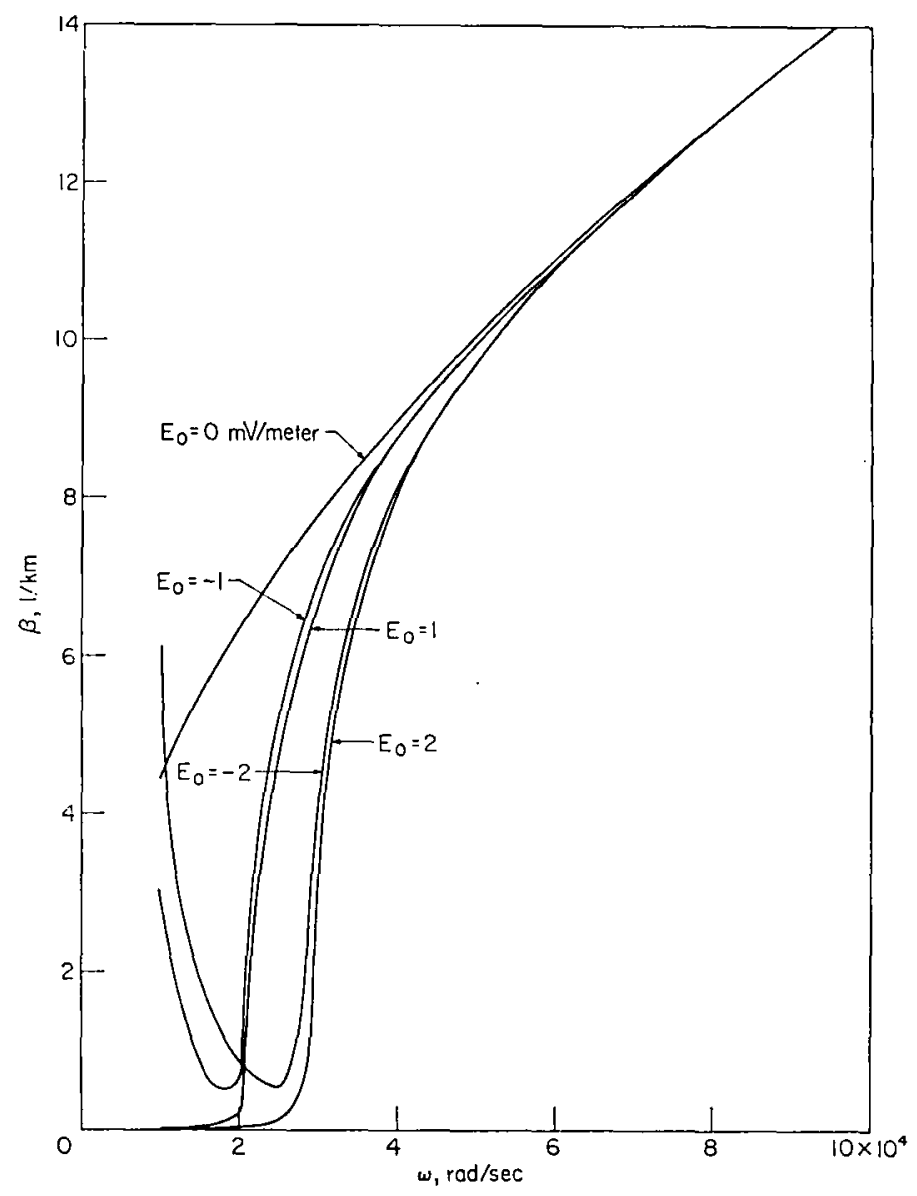

Fig. 2 b. $\beta$ vs. $\omega$ with $E_{0}$ as parameter for Case II.

It is also of interest to note that Figs. 1 and 2 both suggest that the extent of influence of $E_{0}$ on the change in the amplitude, wavelength and phase velocity of the whistler mode under consideration in both Cases I and II is much greater in the low frequency range of the spectrum, e.g., $\omega<6 \times 10^{4} \mathrm{rad} / \mathrm{sec}$, than in the high frequency range, e.g., $\omega>6 \times 10^{4} \mathrm{rad} / \mathrm{sec}$.

\section{Concluding Remarks}

In the present paper it is assumed that the collision frequencies of the electron and positive ions are independent of the particle velocity. The classical AppletonHartree equation used for propagation in the ionosphere has this inherent assumption. Because the electron elastic collision frequency with nitrogen molecules in the atmosphere varies as the square of the electron speed (PHelPS and PACK, 1959) a large discrepancy between classical theory and ionospheric experiments can be expected.

However, SHKaRofsky (1961) has derived a generalized Appleton-Hartree equation applicable to any variation of electron collision frequency with electron 


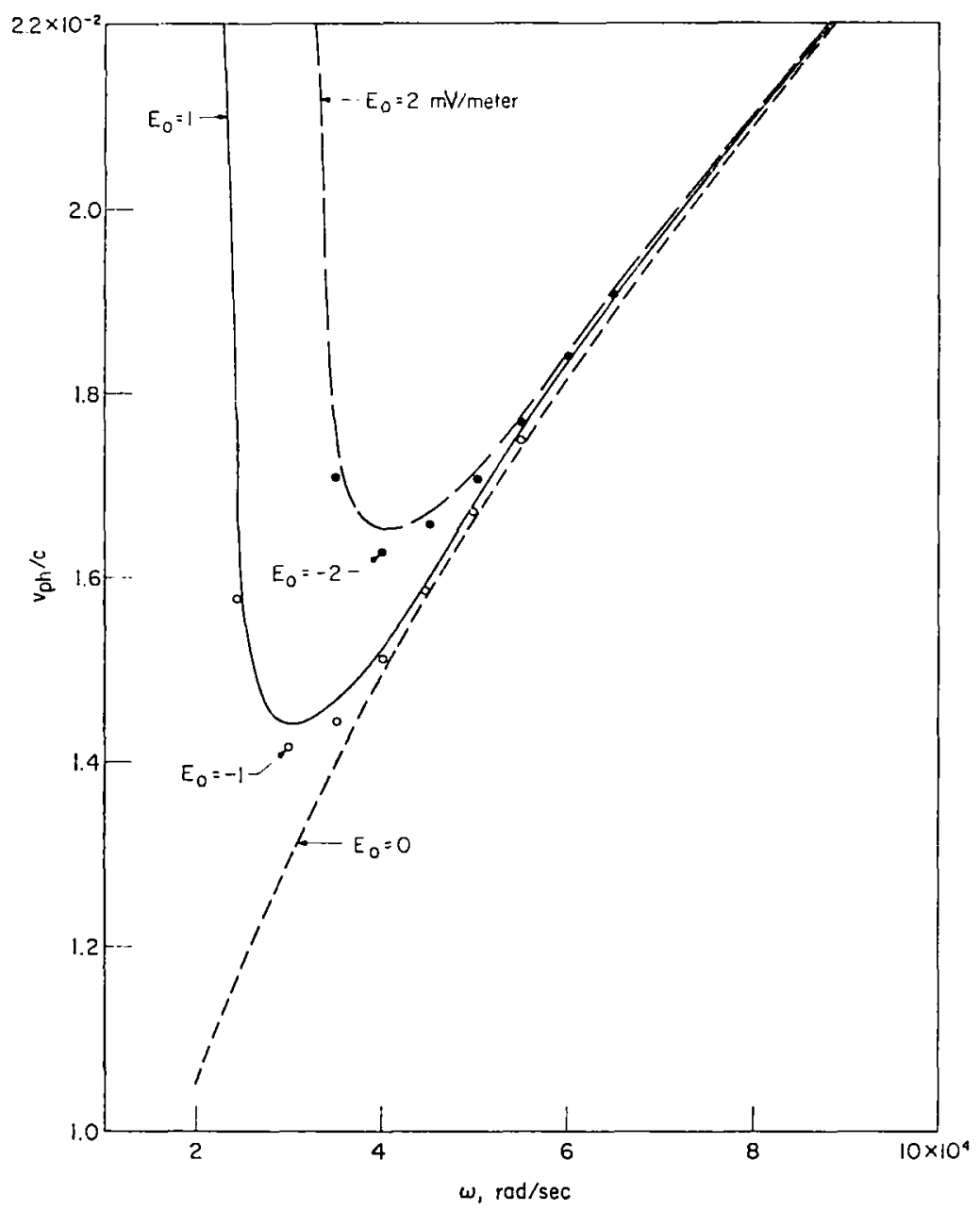

Fig. 2c. $\left(v_{p h} / c\right)$ vs. $\omega$ with $E_{0}$ as parameter for Case II.

speed and any degree of ionization. His analysis suggests that the classical AppletonHartree equation should be applicable with no corrrection necessary for the region of the ionosphere and the range of parameters under consideration in the present paper. Thus the velocity-independent collision frequency model considered here is reasonable.

Most of the uncertainty in the calculation arises from a lack of knowledge of the electrostatic field strength and the collision frequency of positive ions in the region investigated. Consequently the numerical illustration shown in Figs. 1 and 2 only provide information with regard to the order of magnitude and a general behavior of the propagation constant. Although only the forward whistler mode has been considered in these figures, it is not difficult to analyze the propagation characteristic of the backward whistler mode, which is also characterized by equation (2a).

The frequency range used for the illustration lies within the range over which a class of naturally occurring radio noise in the ionosphere and magnetosphere such as a whistler and VLF emission is observed (HELLIWELL, 1965). The result presented in 
this paper should be useful for the study of the propagation of a whistler or VLF emission in the region of ionosphere where the presence of electrostatic field may be of significance.

\section{References}

Ferraro V. C. and Peunipton C.

1961 An Introduction to Magneto-Fluid Mecha-

Helutweld R. A. nics. Clarendon Press, Oxford, England.

HSIEH H. C.

Mozer F. S. and Bruston P. Whistlers and Related Ionospheric Phe. nomena. Stanford University Press, Stanford, Calif.

NICOLET M. J.

. Atmosph. Terr. Phys. (in pross).

SHKAROFSKY I. D.

J. Geophys. Res. 72, 1109.

1967

1967

1953

J. Atmosph. Terr. Phys., 3, 200.

VALLEY S. L.

1961 Proc. I.R.E. 49, 1857.

1965 Handbook of Geophysics and Space Environments, McGraw-Hill, New York, Chap. 12. 